\title{
BMJ Open EUropean prospective cohort study on Enterobacteriaceae showing REsistance to CArbapenems (EURECA): a protocol of a European multicentre observational study
}

Belén Gutiérrez-Gutiérrez, ${ }^{1}$ Jesús Sojo-Dorado, ${ }^{1}$ José Bravo-Ferrer, ${ }^{1}$ Nienke Cuperus, ${ }^{2}$ Marlieke de Kraker, ${ }^{3}$ Tomislav Kostyanev, ${ }^{4,5}$ Lul Raka, ${ }^{6}$ George Daikos, ${ }^{7}$ Jan Feifel, ${ }^{8}$ Laura Folgori, ${ }^{9}$ Alvaro Pascual, ${ }^{1}$ Herman Goossens, ${ }^{4,5}$ Seamus O'Brien, ${ }^{10}$ Marc J M Bonten, ${ }^{2}$ Jesús Rodríguez-Baño, ${ }^{1}$ for the EURECA project team

To cite: Gutiérrez-Gutiérrez B, Sojo-Dorado J, BravoFerrer J, et al. EUropean prospective cohort study on Enterobacteriaceae showing REsistance to CArbapenems (EURECA): a protocol of a European multicentre observational study. BMJ Open 2017;7:e015365. doi:10.1136/bmjopen-2016015365

- Prepublication history for this paper is available online. To view these files please visit the journal online (http://dx.doi.org/10.1136/ bmjopen-2016-015365).

Received 2 December 2016 Revised 6 February 2017 Accepted 7 February 2017

CrossMark

For numbered affiliations see end of article.

Correspondence to Dr Belén Gutiérrez-Gutiérrez; belengutiguti@hotmail.com

\section{ABSTRACT}

Introduction: The rapid worldwide spread of carbapenem-resistant Enterobacteriaceae (CRE) constitutes a major challenge. The aim of the EUropean prospective cohort study on Enterobacteriaceae showing REsistance to CArbapenems (EURECA), which is part of the Innovative Medicines Initiative Joint Undertaking (IMI JU) funded COMBACTE-CARE project, is to investigate risk factors for and outcome determinants of CRE infections to inform randomised clinical trial designs and to provide a historical cohort that could eventually be used for future comparisons with new drugs targeting CRE.

Methods: A multicentre (50 sites), multinational (11 European countries), analytical observational project was designed, comprising 3 studies. The aims of study 1 (a prospective cohort study) include characterising the features, clinical management and outcomes of hospitalised patients with intra-abdominal infection, pneumonia, complicated urinary tract infections and bloodstream infections caused by CRE (202 patients in each group). The main outcomes will be 30-day all-cause mortality and clinical response. Study 2 (a nested case-control study) will identify the risk factors for target infections caused by CRE; 248 selected patients from study 1 will be matched with patients with carbapenem-susceptible Enterobacteriaceae (1:1) and with hospitalised patients (1:3) and will provide a historical cohort of patients with CRE infections. Study 3 (a matched cohort study) will follow patients in study 2 in order to assess mortality, length of stay and hospital costs associated with CRE. All patients will be followed for 30 days. Different, up-to-date statistical methods will be applied to come to unbiased estimates for all 3 studies.

Ethics and dissemination: Before-study sites will be initiated, approval will be sought from appropriate regulatory agencies and local Ethics Committees of Research or Institutional Review Boards (IRBs) to conduct the study in accordance with regulatory

\section{Strengths and limitations of this study}

- The EUropean prospective cohort study on Enterobacteriaceae showing REsistance to CArbapenems (EURECA) is a prospective, multinational (11 European countries), multicentre (50 sites) study with onsite investigators collecting a magnitude of reliable information on individual level as well as hospital level to reliably characterise risk factors, clinical management, best available therapy (BAT) and outcomes of hospitalised patients with four different types of infections caused by carbapenem-resistant Enterobacteriaceae (CRE).

- It will also provide a historical cohort treated with the BAT, enabling inferiority trials as well as historically controlled superiority trials.

- Different up-to-date statistical methods will be applied to come to unbiased estimates for the three studies that EURECA includes.

- This study has limitations, including its mainly observational nature, so an impact of unmeasured variables and residual confounding cannot be discarded. Also, despite the fact that this will be, to the best of our knowledge, one of the biggest cohort of patients with infections caused by CRE, the statistical power in some strata may be limited.

requirements. This is an observational study and therefore no intervention in the diagnosis, management or treatment of the patients will be required on behalf of the investigation. Any formal presentation or publication of data collected from this study will be considered as a joint publication by the participating physician(s) and will follow the recommendations of the International Committee of Medical Journal Editors (ICMJE) for authorship.

Trial registration number: NCT02709408. 


\section{INTRODUCTION}

Antibiotic resistance is recognised as an important global public health concern. ${ }^{1}$ Among antibiotic-resistant organisms, carbapenem-resistant Enterobacteriaceae (CRE) have spread rapidly worldwide and now present a major challenge for treating patients suffering from infections caused by them. Resistance to carbapenems can be mediated by different mechanisms, although the production of carbapenemases ( $\beta$-lactamases with hydrolytic activity against carbapenems) is the most worrisome because of their recent dramatic spread in many countries. $^{2} \quad$ Unfortunately, carbapenemase-producing Enterobacteriaceae (CPE) are frequently resistant to other antibiotic families, which considerably limits the available therapeutic options against them.

Until now, little is known on several aspects of infections due to CRE and/or CPE. First, although a meta-analysis showed that infections caused by CRE were associated with higher mortality rates than those caused by carbapenem-susceptible isolates, ${ }^{3}$ the studies providing the primary data had methodological limitations such as insufficient control for confounders. Second, most of the studies investigating the clinical impact and outcome of these infections were carried out in specific environments (such as intensive care units (ICUs) or outbreaks), or included only specific types of infection. Third, the management patterns in different settings and their impact on clinical outcomes were not defined. Fourth, the currently recommended therapy (use of at least two active drugs, including at least one carbapenem if the minimum inhibitory concentration (MIC) of these drugs is sufficiently low ${ }^{4}$ is based only on retrospective studies, which mostly included bacteraemic infections caused by Klebsiella pneumoniae carbapenemase (KPC)-producing Klebsiella pneumoniae.$^{5-7}$ As a result, the best available therapy (BAT) is not well defined for different organisms or clinical situations. These issues are important in order to evaluate medical needs in this area and to design randomised controlled trials with newer and older drugs.

Performing randomised trials can be particularly difficult in the case of multidrug-resistant organisms (MDRO), because patients cannot be selectively recruited before microbiological results are available and empirical treatment can dilute the impact of the drugs under study. Historical control groups have been viewed as an option for providing rapid real-world data on the efficacy of anti-infectives specifically in the case of serious infections with poor outcomes and limited treatment options, like those caused by CRE. ${ }^{8}$ To be useful for such purposes, the historical cohort should include all known confounders of the effect, use consistent exposure and outcome definitions, and data quality should be ensured.

The EUropean prospective cohort study on Enterobacteriaceae showing REsistance to CArbapenems (EURECA), which is part of the Innovative Medicines Initiative Joint Undertaking (IMI JU)-funded
COMBACTE-CARE project, ${ }^{9}$ aims to clarify some of the above gaps and to support regulatory pathways for new antibiotics. ${ }^{10}$ The main objectives of EURECA are: (1) to identify the predictors for negative outcomes in patients with intra-abdominal infection (IAI), pneumonia, complicated urinary tract infections (cUTI) and bloodstream infections (BSI) caused by CRE, including the impact of clinical management and different antimicrobial regimens on outcome and identification of the BAT; (2) to identify the risk factors for the aforementioned infections caused by CRE; (3) to provide cohorts of patients with the target infections caused by CRE that could eventually be used as historical cohorts for making comparisons of the efficacy and safety of other drugs against CRE organisms; and (4) to assess the mortality, length of hospital stay and hospitalisation costs associated with the target infections caused by CRE.

\section{METHODS AND ANALYSIS \\ Studies and design}

To answer the above questions, a prospective, multinational, multicentre, analytical observational project was designed that included three substudies (figure 1). The study designs are:

Study 1: To achieve objectives 1 and 3, a prospective cohort study will be carried out comprising patients with targeted infections due to CRE.

Study 2: To achieve objective 2, a matched nested case-control study will be performed. Cases will be selected from study 1 . The first group of matched controls will be selected among patients with infections caused by carbapenem-susceptible Enterobacteriaceae (CSE), and the second group of matched controls will comprise admitted patients without CRE infection (admitted patients).

Study 3: To achieve objective 4, a matched cohort study will be conducted. Cohorts will comprise patients with infections due to CRE, patients with infections due to CSE and 'admitted patients' included in study 2.

\section{Sites and study period}

The study will be performed in 50 hospitals throughout countries in southern Europe including Albania, Croatia, Greece, Italy, Kosovo, Montenegro, Romania, Serbia, Spain and Turkey. The participating sites were

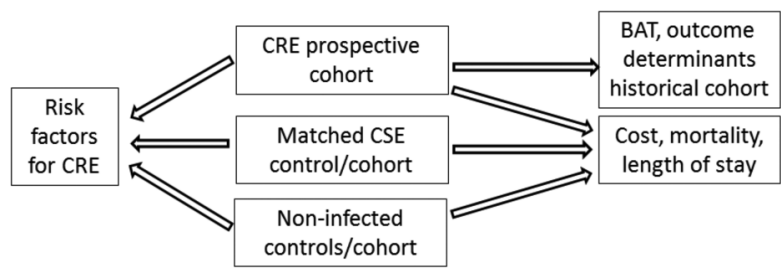

Figure 1 Study design according to targeted objectives. BAT, best available therapy; CRE, carbapenem-resistant Enterobacteriaceae; CSE, carbapenem-susceptible Enterobacteriaceae. 
selected after completing specific feasibility questionnaires that included data on rates of infection due to CRE, number of beds for adult and paediatric patients, clinical and laboratory capabilities and experience in clinical studies.

All consecutive patients with IAI, pneumonia, cUTI and BSI due to CRE diagnosed at the participating hospitals (202 patients in each group) will be eligible. These patients will be identified daily by reviewing local microbiological laboratory reports at each site. Each patient with any Enterobacteriaceae isolate fulfilling the microbiological criteria for CRE (see below) taken from a clinical sample (screening samples will not be considered) will be evaluated for inclusion and exclusion criteria. These patients will form the CRE cohorts in study 1; 248 of these patients will be selected to serve as the CRE case group in study 2 and the CRE cohort in study 3 (figure 2).

For each CRE case patient, one patient will be included from whom CSE has been isolated; CSE controls will be matched by centre, type of acquisition (community or nosocomial), type of hospital service, length of hospitalisation before infection and type of infection. These patients will serve as the matched CSE control group of study 2 and the matched CSE cohort of study 3.

Finally, for each CRE case patient, three admitted patients without Enterobacteriaceae infection will be included matched to the corresponding CRE case by centre, ward and length of hospitalisation. These patients will serve as the matched admitted patient controls in study 2, and as the matched 'admitted patient' cohort in study 3 .

All patients will be followed for a period of 30 days after inclusion. A summary of the three key visits during follow-up is shown in table 1 .

\section{Inclusion criteria for CRE patients}

- CRE is isolated from a clinical sample (eg, a sample obtained in the work-up of a patient with suspicion of infection; hence, screening samples will not be considered).

- The patient meets the criteria for any of the following infections (see definitions below): cUTI, pneumonia, IAI or BSI.

- The patient or his/her representative has signed an informed consent form if requested by the local Institutional Review Board (IRB).

\section{Exclusion criteria for CRE patients}

- The infection is considered to be polymicrobial according to standard microbiological interpretations of culture results (except for IAI, in which polymicrobial infections are allowed).

- The patient is participating in a clinical trial that involves active treatment for these infections.

- The patient was previously included in the same cohort of this study for the same organism. A single episode of CRE per patient may be included.

- Patients with do-not-resuscitate orders or with a life expectancy of $<30$ days.

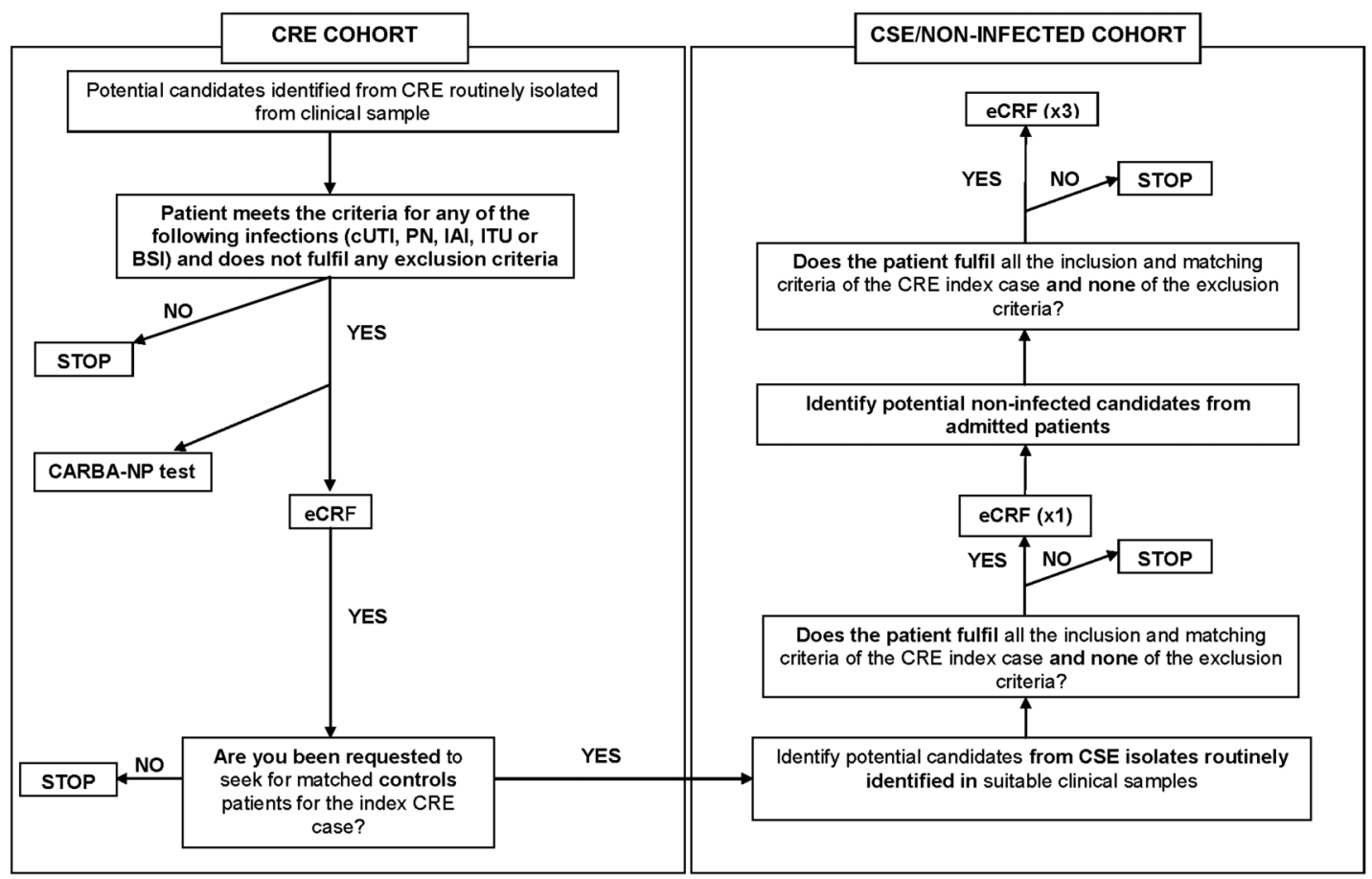

Figure 2 Decision tree for patient enrolment. BSI, blood stream infection; CRE, carbapenem-resistant Enterobacteriaceae; CSE, carbapenem-susceptible Enterobacteriaceae; cUTI, complicated urinary tract infection; eCRF, electronic case report form; IAI, intra-abdominal infection; PN, pneumonia. 


\section{Study variables}

The primary end points for study 1 are 30-day all-cause mortality and clinical response at day 21 (test-of-cure, TOC), categorised as failure versus cure/improvement. The secondary end points are: microbiological response at TOC, mortality during hospitalisation, infectionrelated mortality until day 21, length of hospital stay after infection, length of ICU stay (if appropriate), duration of antibiotic treatment, recurrence, superinfection and therapy-related adverse events.

The primary end point for study 2 is infection due to CRE. The primary end points for study 3 are 30-day allcause mortality, length of hospital stay after infection, length of ICU stay and duration of mechanical ventilation, if appropriate.

The following explanatory variables will be collected: age, gender, date of admission, ethnicity, country, hospital, type of hospital ward, recent travel abroad, contact with pets or livestock, profession, contact with persons colonised by CRE during previous hospitalisations in an acute care hospital or long-term care facility, previous colonisation by CRE, comorbidities (type and severity according to the Charlson index for adults ${ }^{11}$ and Antibiotic Resistance and Prescribing in European Children (ARPEC) Point Prevalence Survey (ARPEC PPS) definitions for children), ${ }^{12}$ transplantation, neutropaenia, use of immunosuppressive drugs, HIV infection with $<200 \mathrm{CD} 4$ cells $/ \mathrm{mm}^{3}$ type of acquisition (nosocomial, healthcare-associated or community), ${ }^{13}{ }^{14}$ severity of sepsis, ${ }^{15}{ }^{16}$ Pitt score, ${ }^{17}$ Sepsis-related Organ Failure Assessment (SOFA) and QuickSOFA ${\text { scores },{ }^{18} 19}^{19}$ Pediatric Early Warning Score (PEWS) score in children, ${ }^{20}$ Acute Physiology And Chronic Health Evaluation II (APACHE II) score in ICU patients, ${ }^{21}$ Pediatric Index of Mortality 2 (PIM2)2 in children admitted to the ICU, ${ }^{22}$ invasive procedures (mechanical ventilation, central venous catheter, urinary catheter, surgery), type of infection, microbiological features of the isolate (carbapenemase producer or not, carbapenemase type, susceptibility profile, carbapenem MICs), clinical management other than antimicrobial therapy (source control, support therapy), antimicrobial therapy (including all antibiotics administered, dose, start and discontinuation dates, reason/s for discontinuation) and adverse events related to antimicrobial therapy (renal toxicity, liver toxicity, Clostridium difficile infection).

\section{Definitions}

The definitions of cUTI, pneumonia, IAI and BSI are shown in table 2. For the purpose of the study, a CRE is defined as any isolate identified as an Enterobacteriaceae showing a MIC of meropenem or imipenem of $\geq 1 \mathrm{mg} / \mathrm{L}$ if using any dilution method, and/or of $\leq 22 \mathrm{~mm}$ if using a disk-diffusion method (10 $\mu \mathrm{g}$ disks). A CSE is defined as any isolate identified as an Enterobacteriaceae showing susceptibility to carbapenems according to the above criteria. Meropenem-susceptible and imipenemsusceptible isolates showing resistance to ertapenem will be excluded.

\section{Microbiological studies}

Local laboratories will identify the isolates and perform routine susceptibility testing according to standard microbiological procedures; also, CRE isolates will be studied locally using the CARBA NP test. All CRE isolates will be preserved at $-20^{\circ} \mathrm{C}$ and sent to a central laboratory for detection and characterisation of carbapenemase genes.

\section{Sample size}

The sample size for the CRE has been calculated in a way that the cohorts can serve as 'historical' cohorts in future comparisons involving other drugs against CRE infections. To do this, and because the estimate for the outcome variable of the new drug is unknown, we seek to generate clinical cure rate estimates for the BAT with 95\% CI and $8 \%$ precision. For an estimated cure rate of $50 \%$, based on data from previous studies, ${ }^{5-7} 151$ patients with each type of infection (cUTI, pneumonia, IAI and BSI) are needed. However, since $\sim 25 \%$ of patients will not receive the BAT, 201 patients per type of infection will be included. This sample size will also

Table 1 Simplified follow-up schedule and variables to be collected

\begin{tabular}{lllll}
\hline Variables & Day 0 & From day 0 to 21 & Day 21 (TOC) & Day 30 (end of follow-up) \\
\hline Selection criteria & $\sqrt{ }$ & & & \\
Demographics & $\sqrt{ }$ & & & \\
Risk factors & $\sqrt{ }$ & & $\sqrt{ }$ & \\
Comorbidities & $\sqrt{ }$ & $\sqrt{ }$ & $\sqrt{ }$ & $\sqrt{ }$ \\
Clinical features & $\sqrt{ }$ & $\sqrt{ }$ & $\sqrt{ }$ & $\sqrt{ }$ \\
Microbiology & $\sqrt{ }$ & $\sqrt{ }$ & $\sqrt{ }$ & $\sqrt{ }$ \\
Antimicrobial therapy & $\sqrt{ }$ & $\sqrt{ }$ & \\
Non-antibiotic treatment & $\sqrt{ }$ & $\sqrt{ }$ & & \\
Outcome & & & &
\end{tabular}


Table 2 Definitions of infections included in the study

\begin{tabular}{|c|c|c|}
\hline Type of infection & Definition & \\
\hline $\begin{array}{l}\text { Complicated } \\
\text { urinary tract } \\
\text { infection }\end{array}$ & $\begin{array}{l}\text { For adults and children }>12 \text { years (any of them) } \\
\text { A positive blood culture for CRE or CSE } \\
\text { and } \\
\text { One of the following: } \\
\text { One local symptom (urgency, frequency, } \\
\text { dysuria, tenesmus or suprapubic tenderness) } \\
\text { or } \\
\text { Two systemic symptoms or predisposing } \\
\text { conditions (fever ( }>38^{\circ} \mathrm{C} \text { core or }>38.3^{\circ} \mathrm{C} \\
\text { armpit) or hypothermia }\left(<36^{\circ} \mathrm{C} \text { core), new }\right. \\
\text { cognitive impairment or change in mental status } \\
\text { (in patients over } 70 \text { years), flank pain, } \\
\text { costovertebral angle tenderness on physical } \\
\text { examination, urinary tract abnormalities or } \\
\text { presence of a urinary catheter) } \\
\text { or } \\
\text { A positive urine culture (isolation of CRE or } \\
\text { CSE, } \geq 10^{5} \text { microorganisms per mL of urine) } \\
\text { and } \\
\text { No other recognised cause of the bloodstream } \\
\text { infection } \\
\text { For children }>2 \text { years } \\
\text { Abnormal urinary dipstick test (leucocyte esterase } \\
\text { centrifuged urine, and bacteriuria with any bacteri } \\
\text { and } \\
\text { At least two of the following clinical or biological s } \\
\text { 1. Fever with temperature of } 38^{\circ} \mathrm{C} \text { or higher } \\
\text { 2. Abdominal or flank pain, urgency, frequency, } \\
3 \text {. Elevated } \mathrm{C} \text { reactive protein or procalcitonin co } \\
\text { and } \\
\text { A positive urine culture with isolation of CRE or C } \\
\text { aspirate/urinary catheter with } \geq 10^{4} \text { microorganism } \\
\text { For children } \leq 2 \text { years old } \\
\text { Abnormal urinary dipstick test (leucocyte esterase } \\
\text { centrifuged urine, and bacteriuria with any bacteri } \\
\text { and } \\
\text { At least two of the following clinical or biological s } \\
1 \text {. Fever with temperature of } 38^{\circ} \mathrm{C} \text { or higher } \\
2 \text {. General, non-specific signs such as irritability, } \\
3 \text {. Elevated } \mathrm{C} \text { reactive protein or procalcitonin co } \\
\text { and }\end{array}$ & $\begin{array}{l}\text { >1+, or positive nitrite test) or urinalysis (pyuria with at least } 10 \text { WCCs per high power field in } \\
\text { aper high power field on an unstained specimen of urinary sediment) } \\
\text { gns: } \\
\text { ysuria, suprapubic tenderness } \\
\text { ncentrations, according to the local laboratory } \\
\text { S only (spontaneously voided urine with } \geq 10^{5} \text { microorganisms per } \mathrm{mL} \text { of urine or suprapubic } \\
>1+\text { or positive nitrite test) or urinalysis (pyuria with at least } 10 \text { WCCs per high power field in } \\
\text { a per high power field on an unstained specimen of urinary sediment) } \\
\text { gns: } \\
\text { vomiting, diarrhoea or feeding problems in infants } \\
\text { ccentrations according to the local laboratory }\end{array}$ \\
\hline
\end{tabular}

\section{Type of infection} A positive blood culture for CRE or CSE
and

One of the following:

One local symptom (urgency, frequency, dysuria, tenesmus or suprapubic tenderness) (in patients over 70 years), flank pain, costovertebral angle tenderness on physical presence of a urinary catheter) CSE, $\geq 10^{5}$ microorganisms per $\mathrm{mL}$ of urine) and infection

For children >2 years centrifuged urine, and bacteriuria with any bacteria per high power field on an unstained specimen of urinary sediment)

1. Fever with temperature of $38^{\circ} \mathrm{C}$ or higher

2. Abdominal or flank pain, urgency, frequency, dysuria, suprapubic tenderness and aspirate/urinary catheter with $\geq 10^{4}$ microorganisms per $\mathrm{mL}$ of urine) or a positive blood culture with isolation of CRE with no other recognised cause For children $\leq 2$ years old centrifuged urine, and bacteriuria with any bacteria per high power field on an unstained specimen of urinary sediment)

2. General, non-specific signs such as irritability, vomiting, diarrhoea or feeding problems in infants and 
A positive urine culture with isolation of CRE or CSE only (spontaneously voided urine with $\geq \mathrm{Ab} 10^{5}$ microorganisms per $\mathrm{mL}$ of urine or suprapubic

Intra-abdominal

infection

Pneumonia aspirate/urinary catheter with $\geq 10^{4}$ microorganisms per $\mathrm{mL}$ of urine) or positive blood culture with isolation of CRE with no other recognised cause For all age groups (either of them)

Patient has organisms cultured from purulent material from intra-abdominal space obtained during a surgical operation or needle aspiration.
Patient has at least two of the following signs or symptoms with no other recognised cause:

1. Fever $\left(>38^{\circ} \mathrm{C}\right)$

2. Nausea

3. Vomiting

4. Abdominal pain

5. Jaundice

and

One of the following:

1. Organisms cultured from drainage from surgically placed drain (eg, closed suction drainage system, open drain, T-tube drain)

2. Organisms cultured from blood and radiographic evidence of infection, for example, abnormal findings on ultrasound, CT scan, MRI or radiolabelled scans (gallium, technetium, etc) or on abdominal X-ray

For all age groups (at least one of the following criteria: radiological, clinical and microbiological) Radiology

Chest X-rays or CT scan with a suggestive image of pneumonia (for patients with Clinical criteria

For children $\leq 12$ years oldAt least three of the following:

underlying cardiac or pulmonary disease, a new 1 . Fever $>38^{\circ} \mathrm{C}$ with no other cause

infiltrate needs to be demonstrated by comparing 2. Leucocytosis or leucopaenia (see

with a previous chest X-ray or CT scan) age-specific laboratory variables above)

3. Worsening gas exchange $\left(\mathrm{eg}, \mathrm{O}_{2}\right.$ desaturations (eg, pulse oximetry reading $<94 \%$ ), increased oxygen requirements or increased ventilator demand)

4. Apnoea, tachypnoea, nasal flaring with retraction of chest wall or grunting

5. Wheezing, rales or rhonch

6. Cough

7. Bradycardia or tachycardia (age-specific) For children $\geq 12$ and adults

At least one each of the following symptoms/ signs/laboratory data:

1. Fever $>38^{\circ} \mathrm{C}$

2. Leucocytosis $\left(\geq 12000 \mathrm{WCC} / \mathrm{mm}^{3}\right)$

3. Leucopaenia $\left(<4000 \mathrm{WCC} / \mathrm{mm}^{3}\right)$

4. In patients $\geq 70$ years old, new cognitive impairment or worsening mental status

\section{Microbiology}

Isolation of bacteria from any of the following:

1. Quantitative culture from minimally

contaminated lower respiratory tract specimen (bronchoalveolar lavage (BAL) with a threshold of $>10^{4} \mathrm{CFU} / \mathrm{mL}$ or $\geq 5 \%$ of BAL-obtained cells containing intracellular bacteria on direct microscopic examination; protected specimen brush with a threshold of $>10^{3} \mathrm{CFU} / \mathrm{mL}$; distal protected aspirate with a threshold of $>10^{3}$ $\mathrm{CFU} / \mathrm{mL}$ )

2. Quantitative culture of endotracheal aspirate or unprotected specimen brush with a threshold of $10^{6} \mathrm{CFU} / \mathrm{mL}$

3. Blood cultures, not related to any other source of infection

4. Pleural fluid or needle aspiration of pleural or pulmonary abscess

5. Sputum culture with quality criteria $(>25$ leucocytes/field 100X and $<10$ squamous epithelial cells/field 100X) 

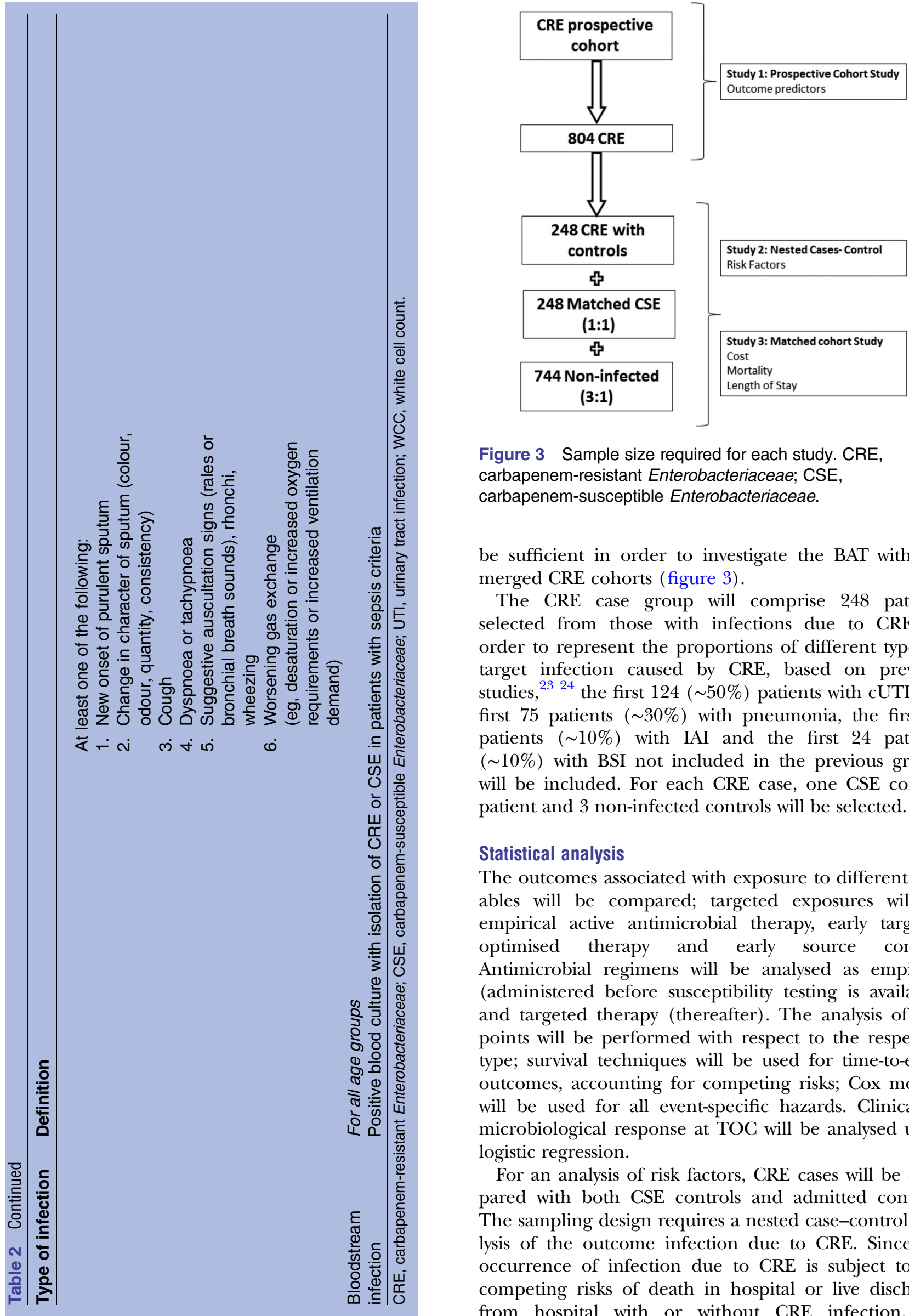

Figure 3 Sample size required for each study. CRE, carbapenem-resistant Enterobacteriaceae; CSE, carbapenem-susceptible Enterobacteriaceae.

be sufficient in order to investigate the BAT with the merged CRE cohorts (figure 3).

The CRE case group will comprise 248 patients selected from those with infections due to CRE. In order to represent the proportions of different types of target infection caused by CRE, based on previous studies, ${ }^{23}{ }^{24}$ the first $124(\sim 50 \%)$ patients with cUTI, the first 75 patients $(\sim 30 \%)$ with pneumonia, the first 25 patients $(\sim 10 \%)$ with IAI and the first 24 patients $(\sim 10 \%)$ with BSI not included in the previous groups will be included. For each CRE case, one CSE control patient and 3 non-infected controls will be selected.

\section{Statistical analysis}

The outcomes associated with exposure to different variables will be compared; targeted exposures will be empirical active antimicrobial therapy, early targeted optimised therapy and early source control. Antimicrobial regimens will be analysed as empirical (administered before susceptibility testing is available) and targeted therapy (thereafter). The analysis of end points will be performed with respect to the respective type; survival techniques will be used for time-to-event outcomes, accounting for competing risks; Cox models will be used for all event-specific hazards. Clinical or microbiological response at TOC will be analysed using logistic regression.

For an analysis of risk factors, CRE cases will be compared with both CSE controls and admitted controls. The sampling design requires a nested case-control analysis of the outcome infection due to CRE. Since the occurrence of infection due to CRE is subject to the competing risks of death in hospital or live discharge from hospital with or without CRE infection, the 
analyses will be supplemented with those of the competing outcomes. Multilevel hospital data (local CRE rate, antimicrobial consumption, infection control measures) will also be considered in the analyses above.

Mortality, length of hospital and ICU stay, and duration of mechanical ventilation of patients with CRE, CSE and admitted patients will be compared. All time-to-event outcomes will be compared using survival techniques. Extra hospital/ICU days will be estimated using Beyersmann et $a l \mathrm{~s}^{25}$ multistate approach. Goodness of fit will be assessed, and variable selection will be based on Akaike's information criterion ${ }^{26}$ throughout.

\section{ETHICAL ISSUES AND DISSEMINATION PLAN}

This study will be conducted according to the principles of the Declaration of Helsinki and in accordance with the Medical Research Involving Human Subjects Act (WMO) and local guidelines in the participating countries. Before-study sites will be initiated, approval will be sought from appropriate regulatory agencies and local Ethics Committees of Research or IRBs to conduct the study in accordance with regulatory requirements. This is an observational study and therefore no intervention in the diagnosis, management or treatment of the patients will be required on behalf of the investigation. All patient management, including all antibiotic regimens prescribed, will be decided by the physician/team in charge without any interference. No management procedures from routine evidence-based management are included as part of the investigation.

Processing of the patients' personal data collected in this study will comply with the Data Protection Act 1998 and the European Directive on the Privacy of Data. All data collected, stored and processed will be anonymised (95/46/EC). The principal investigator/lead researcher at each site will guarantee that all team members or other persons involved at his site respect the confidentiality of any information concerning the study patients and ensure that the personal privacy of any patient whose data are collected in the study is not violated.

Individual written consent of the patient is not required; however, if any local or central IRB decides that written informed consent is needed, it will be required at the specific sites. Training in research methods for the study will be provided for all site personnel involving face-to-face meetings and webcast teleconferences. To guarantee data quality and avoid fraud, the study sites will be monitored and/or audited. The sponsor or a designated third party will conduct monitoring and audit visits.

Spot check monitoring and source data verification will be conducted to identify that patients exist and that the data entered in the database have been retrieved from genuine patient files and transferred correctly. All site study-related documents, including patient data source documents, must be made available for monitoring and audit.
Results will be reported at conferences and in peerreviewed publications. It is mandatory that any publication is based on data from the database, analysed as stipulated in the protocol by investigators in agreement with the sponsor.

Any formal presentation or publication of data collected from this study will be considered as a joint publication by the participating physician(s) and will follow the recommendations of the International Committee of Medical Journal Editors (ICMJE) for authorship.

\section{DISCUSSION}

While antimicrobial resistance is recognised as an important health problem worldwide, it is challenging to perform high-quality comparative clinical research on therapeutic options for infections caused by antimicrobial-resistant bacteria. The reported outcomes of infections caused by MDROs are usually worse than those caused by susceptible organisms, but appropriate control of all relevant confounders is key because MDROs frequently affect debilitated patients prone to worse prognoses, and this is especially true for CRE cases. Appropriateness of therapy, an intermediate variable, should be considered as well, but for CRE cases the BAT is unknown. Then hospital-level variables can greatly influence clinical outcomes as well, and therefore it is of paramount importance to measure risk factors and clinical outcomes in different environments: higher versus lower endemicity, small versus large hospitals, large infection control bundles versus smaller bundles. Therefore, EURECA is a prospective, multinational, multicentre study with onsite investigators collecting a magnitude of reliable information on individual level as well as hospital level to reliably characterise risk factors, clinical management, BAT and outcomes of hospitalised patients with four different types of CRE infections.

On the basis of the results of retrospective cohort studies, mainly including patients with bacteraemic infections caused by KPC-producing Klebsiella pneumoniae, it has been suggested that the use of combination therapy is superior to monotherapy for severe/invasive infections caused by CPE. ${ }^{5-7}$ Nevertheless, the BAT for different types of infections and different types of patients in different settings remains ambiguous. EURECA will be able to inform future clinical trials on anti-infectives against CRE infections about the BAT. Moreover, it will enable researchers to efficiently include a high proportion of CRE cases, based on clinical profile, and to decide on appropriate sample sizes, based on detailed clinical outcome estimates for different settings. It will also provide a historical cohort treated with the BAT, enabling inferiority trials as well as historically controlled superiority trials. ${ }^{8}$

To the best of our knowledge, despite the fact that the reported mortality rates of patients with infections due to CRE are frequently high, the clinical pathway is unclear and evidence of excess mortality is limited. ${ }^{3}$ 
Possible mechanisms responsible for negative outcomes include delay in active therapy, use of substandard drugs and/or increased virulence of CRE. However, in evidence-based medicine, it is crucial to produce reliable, actionable information so that quality of care in these complicated cases can be improved.

In conclusion, EURECA aims to provide actionable information for the design of randomised control trials for the treatment of CRE, as well as robust conclusions for appropriate clinical decision-making until the results of such trials are available.

\author{
Author affiliations \\ ${ }^{1}$ Instituto de Biomedicina de Sevilla, IBiS—Unidad de Gestión Clínica de \\ Enfermedades Infecciosas y Microbiología, Hospital Universitario Virgen \\ Macarena and Departamento de Medicina, Universidad de Sevilla, Seville, \\ Spain \\ ${ }^{2}$ University Medical Center Utrecht, Utrecht, The Netherlands \\ ${ }^{3}$ Infection Control Program, Geneva University Hospitals, Genève, Switzerland \\ ${ }^{4}$ Faculty of Medicine, Department of Medical Microbiology, University of \\ Antwerp, Antwerp, Belgium \\ ${ }^{5}$ Vaccine \& Infectious Disease Institute, University of Antwerp, Antwerp, \\ Belgium \\ ${ }^{6}$ National Institute of Public Health of Kosovo and Faculty of Medicine, \\ University of Prishtina, Prishtina, Serbia \\ ${ }^{7}$ National and Kapodistrian University of Athens, Laikon General Hospital, \\ Athens, Greece \\ ${ }^{8}$ Institute of Statistics, Ulm University, Ulm, Germany \\ ${ }^{9}$ Paediatric Infectious Diseases Research Group, St George's University of \\ London, London, UK \\ ${ }^{10}$ AstraZeneca, Cheshire, UK
}

Acknowledgements Other members of the EURECA project team: Lorena López-Cerero, Almudena Serna, Sophie Monteau and Virginia Palomo (Hospital Universitario Virgen Macarena), Perlat Kapisyzi and Silva Tafaj (University Hospital of Lung Diseases 'Shefqet Ndroqi'), Patricia Muñoz (Hospital Universitario Gregorio Marañón), Adriana Vince and Arjana Tambic Andrasevic (University Hospital for Infectious Diseases, Zagreb), Arsim Kurti (National Institute of Public Health of Kosovo), Gazmend Spahija (University Clinical Centre of Kosovo), Evdoxia Tsigou and Theodora Biniari (Agioi Anargyroi General Hospital of Athens), Sotirios Tsiodras and Loukia Zerva (Attikon University General Hospital), Athanasios Skoutelis and Efi Perivolioti (Evangelismos General Hospital of Athens), Apostolos Komnos and Ioanna Voulgaridi (General Hospital of Larissa), Simeon Metallidis and Efthimia Protonotariou (General University Hospital of Thessaloniki 'Ahepa'), Emmanuel Roilides and Aggeliki Karyoti (Hippokration Hospital of Thessaloniki), Matthew E Falagas and Nicholas Legakis (laso General Hospital), Stavroula Smilakou (Laiko General Hospital), Efstratios Maltezos and Maria Panopoulou (University Hospital of Alexandroupolis), G Dalekos and Efthymia Petinaki (University Hospital of Larissa), Charalampos Gogos and Iris Spiliopoulou (University Hospital of Patras), Alessandro Bartoloni and Gian Maria Rossolini (Florence University Hospital), Fabio Franzetti and Sara Rimoldi (Hospital Luigi Sacco), Francesco Blasi and Milena Arghittu (IRCCS Fondazione Ca Granda Ospedale Maggiore Policlinico), Francesco Giuseppe De Rosa and Rossana Cavallo (Molinette Teaching Hospital), Nicola Petrosillo and Antonio Dicaro (National Institute for Infectious Diseases Lazzaro Spallanzani), PierLuigi Viale and Maria Paola Landini (Policlinico Sant'Orsola Malpighi), Massimo Antonelli and Teresa Spanu (Policlinico Universitario Agostino Gemelli), Claudio Viscoli and Anna Marchese (San Martino University Hospital), Stefano Aliberti and Annalisa Cavallero (University of Milan-Bicocca, San Gerardo), Emanuele Durante Mangoni and Susanna Cuccurullo (University of Campania 'Luigi Vanvitelli'/Monaldi Hospital), Andrei Vata and Olivia Dorneanu (Clinical Hospital Of Infectious Diseases of lasi) Mihaela Lupse and Mirela Flonta (Cluj Napoca Infectious Diseases Clinical Hospital), Corneci Dan and Mariana Buzea (Elias University Emergency Hospital), Dana Tomescu and Camelia Ghita (Fundeni Clinical Hospital), Simin
Aysel Florescu and Maria Nica (Infectious and Tropical Diseases Hospital 'Dr Victor Babes'), Anca Georgescu and Edit Székely (Mures County Clinical Emergency Hospital), Adriana Hristea, Serban Benea and Daniela Talapan (the National Institute of Infectious Diseases Matei Bals), Biljana Carevic and Snezana Jovanovic (Clinical Center of Serbia), Lili Radulović and Natasa Miljkovic (Zvezdara University Medical Center), Ljiljana Bukarica, Vladimir Zivanovic and Teodora Vitorovic (Clinical Center of 'Dragisa Misovic'), Gorana Dragovac and Deana Medic (Institute of Public Health of Vojvodina), Natasa Lukic and Branislava Kocic (Clinical Center Nis), Mileva Mašanovic (Clinical Center of Montenegro), Milena Lopicic and Gordana Mijovic (Institute of Public Health, Podgorica, Montenegro), Evelyn Shaw and Fe Tubau (Hospital Universitari de Bellvitge), José $\mathrm{M}^{\mathrm{a}}$ Aguado and Fernando Chaves (Hospital Universitario 12 de Octubre), Juan de Dios Colmenero and Begoña Palop (Hospital Universitario Carlos Haya), José Ramón Arribas and Julio García (Hospital Universitario La Paz), Vicente Pintado and Rafael Cantón (Hospital Universitario Ramón y Cajal), Julián de la Torre Cisneros and Irene Gracia Ahufinger (Maimonides Biomedical Research Institue of Cordoba (IMIBIC). Reina Sofia University Hospital. University of Córdoba.), Angel Asensio [Hospital Puerta de Hierro and University Francisco de Vitoria (UFV)] and Francisca Portero (Hospital Puerta de Hierro), Alpay Azap and Ceren Karahan (Ankara University), Murat Akova and Banu Sancak (Hacettepe University School of Medicine), Cenk Kirakli and Can Bicmen (Izmir Chest Diseases and Surgery Training and Research Hospital), Volkan Korten and Ufuk Hasdemir (Marmara University School of Medicine), Halis Akalin and Cüneyt Ozakin (Uludag University), Stephan Harbarth and Carolina Fankhauser (Geneva University Hospitals), Christine Lammens (University of Antwerp), Jan Beyersmann (UIm University), Mike Sharland and Julia Bielicki (St George's, University of London), Lionel Tan (GSK) and Anne Thérèse Witschi (Basilea Pharmaceutica International).

Contributors JR-B, BG-G and JS-D were responsible for formulating the overall research questions and for the methodological design of the study, including a preliminary statistical analysis plan, and wrote the first draft of the manuscript. MdK and JF are responsible for the review of the statistical analysis plan of the study and also collaborated in the methodological aspects of the study. JR-B, MB, HG, SO, NC, JB-F, BG-G and TK collaborated in site selection. JR-B is the coordinating investigator and leader of the Coordination Team. TK, HG and AP contributed in all the microbiological details of the study. LF contributed in the inclusion and definition of the paediatric variables. $\mathrm{LR}, \mathrm{GD}, \mathrm{SO}$ and $\mathrm{MB}$ participated in its design and supervised the project. All authors read and approved the final manuscript.

Funding The EURECA project is funded by the Innovative Medicine Initiative (IMI), European Union's Seventh Framework Programme (FP7/2007-2013) and EFPIA companies' in-kind contribution (COMBACTE-CARE, grant agreement 115620 ). JR-B and AP received funding for research from the Spanish Network for Research in Infectious Diseases (REIPI RD12/0015 and RD16/2016), Instituto de Salud Carlos III, MInisterio de Economía y Competitivad, co-financed by the European Development Regional Fund 'A Way to Achieve Europe' ERDF; BG-G is the recipient of a Río Hortega grant from Instituto de Salud Carlos III (CM 15/00265).

Competing interests JR-B has been a scientific advisor for AstraZeneca, Merck and InfectoPharm, and speaker at accredited educational courses supported by Merck. AP has been a speaker for Merck and B Braun; he has been a scientific advisor for Merck and has received unrestricted research grants from B Braun and Astra Zeneca. MJMB has been a speaker for Pfizer. SO is an employee of AstraZeneca.

Ethics approval Before-study sites will be initiated, approval will be sought from appropriate regulatory agencies and local Ethics Committees of Research or Institutional Review Boards (IRBs) to conduct the study in accordance with regulatory requirements.

Provenance and peer review Not commissioned; externally peer reviewed.

Open Access This is an Open Access article distributed in accordance with the Creative Commons Attribution Non Commercial (CC BY-NC 4.0) license, which permits others to distribute, remix, adapt, build upon this work noncommercially, and license their derivative works on different terms, provided the original work is properly cited and the use is non-commercial. See: http:// creativecommons.org/licenses/by-nc/4.0/ 


\section{REFERENCES}

1. World Health Organization (WHO). Antimicrobial resistance: global report on surveillance, 2014. http://www.who.int/drugresistance/ documents/surveillancereport/en/ (accessed 23 Jun 2016).

2. Tzouvelekis LS, Markogiannakis A, Psichogiou M, et al. Carbapenemases in Klebsiella pneumoniae and other Enterobacteriaceae: an evolving crisis of global dimensions. Clin Microbiol Rev 2012;25:682-707.

3. Falagas ME, Tansarli GS, Karageorgopoulos DE, et al. Deaths attributable to carbapenem-resistant Enterobacteriaceae infections. Emerg Infect Dis 2014;20:1171-5.

4. Rodríguez-Baño J, Cisneros JM, Cobos-Trigueros $\mathrm{N}$, et al. Diagnosis and antimicrobial treatment of invasive infections due to multidrug-resistant Enterobacteriaceae. Guidelines of the Spanish Society of Infectious Diseases and Clinical Microbiology. Enferm Infecc Microbiol Clin 2015;33:337.e1-337.e21.

5. Tumbarello M, Viale $\mathrm{P}$, Viscoli $\mathrm{C}$, et al. Predictors of mortality in bloodstream infections caused by KPC-producing Klebsiella pneumoniae: importance of combination therapy. Clin Infect Dis 2012;55:943-50.

6. Qureshi ZA, Paterson DL, Potoski BA, et al. Treatment outcome of bacteremia due to KPC-producing Klebsiella pneumoniae: superiority of combination antimicrobial regimens. Antimicrob Agents Chemother 2012;56:2108-13.

7. Daikos GL, Tsaousi S, Tzouvelekis LS, et al. Carbapenemase-producing Klebsiella pneumoniae bloodstream infections: lowering mortality by antibiotic combination schemes and the role of carbapenems. Antimicrob Agents Chemother 2014;58:2322-8.

8. Infectious Diseases Society of America (IDSA). White paper: recommendations on the conduct of superiority and organism-specific clinical trials of antibacterial agents for the treatment of infections caused by drug-resistant bacterial pathogens. Clin Infect Dis 2012;55:1031-46.

9. Kostyanev T, Bonten MJ, O'Brien S, et al. Innovative medicines initiative's new drugs for bad bugs programme: European public-private partnerships for the development of new strategies to tackle antibiotic resistance. J Antimicrob Chemother 2016;71:290-5

10. Rex JH, Eisenstein $\mathrm{BI}$, Alder $\mathrm{J}$, et al. A comprehensive regulatory framework to address the unmet need for new antibacterial treatments. Lancet Infect Dis 2013;13:269-75.

11. Charlson ME, Pompei $\mathrm{P}$, Ales $\mathrm{KL}$, et al. A new method of classifying prognostic comorbidity in longitudinal studies: development and validation. J Chronic Dis 1987:40:373-83.

12. Versporten A, Bielicki J, Drapier N, et al. The Worldwide Antibiotic Resistance and Prescribing in European Children (ARPEC) point prevalence survey: developing hospital-quality indicators of antibiotic prescribing for children. J Antimicrob Chemother 2016;71:1106-17.
13. Garner JS, Jarvis WR, Grace Emori T, et al. CDC definitions for nosocomial infections. Am J Infect Control 1988;13:128-40.

14. Friedman ND, Kaye KS, Stout JE, et al. Health care-associated bloodstream infections in adults: a reason to change the accepted definition of community-acquired infections. Ann Intern Med 2002;137:791-7.

15. American College of Chest Physicians/Society of Critical Care Medicine: Consensus Conference: definitions for sepsis and organ failure and guidelines for the use of innovative therapies in sepsis. Crit Care Med 1992;20:864-74.

16. Goldstein B, Giroir B, Randolph A. International Consensus Conference on Pediatric Sepsis. International pediatric sepsis consensus conference: definitions for sepsis and organ dysfunction in pediatrics. Pediatr Crit Care Med 2005;6:2-8.

17. Rhee JY, Kwon KT, Ki HK, et al. Scoring systems for prediction of mortality in patients with intensive care unit-acquired sepsis: a comparison of the Pitt bacteremia score and the Acute Physiology and Chronic Health Evaluation II scoring systems. Shock 2009;31:146-50.

18. Vincent JL, Moreno R, Takala J, et al. The SOFA (Sepsis-related Organ Failure Assessment) score to describe organ disfunction failure. On behalf of the Working Group on Sepsis-Related Problems of the European Society of Intensive Care Medicine. Intensive Care Med 1996;22:707-10.

19. Singer M, Deutschman CS, Seymour CW, et al. The third international consensus definitions for sepsis and septic shock (Sepsis-3). JAMA 2016;315:801-10.

20. Duncan $\mathrm{H}$, Hutchison J, Parshuram CS. The pediatric early warning system score: a severity of illness score to predict urgent medical need in hospitalized children. J Crit Care 2006;21:271-8.

21. Knaus WA, Draper EA, Wagner DP, et al. APACHE II: a severity of disease classification system. Crit Care Med 1985;13:818-29.

22. Slater A, Shann F, Pearson G. PIM2: a revised version of the Paediatric Index of mortality. Intensive Care Med 2003;29:278-85.

23. Tumbarello M, Trecarichi EM, De Rosa FG, et al. Infections caused by KPC-producing Klebsiella pneumoniae: differences in therapy and mortality in a multicentre study. J Antimicrob Chemother 2015;70:2133-43.

24. Palacios-Baena ZR, Oteo J, Conejo C, et al. Comprehensive clinical and epidemiological assessment of colonisation and infection due to carbapenemase-producing Enterobacteriaceae in Spain. J Infect 2016;72:152-60.

25. Beyersmann J, Wolkewitz M, Allignol A, et al. Application of multistate models in hospital epidemiology: advances and challenges. Biom J 2011;53:332-50.

26. Akaike $\mathrm{H}$. A new look at the statistical model identification. IEEE Trans Automatic Control 1974;19:716-23. 
Correction: EUropean prospective cohort study on

Enterobacteriaceae showing REsistance to CArbapenems

(EURECA): a protocol of a European multicentre

observational study.

Gutiérrez-Gutiérrez B, Sojo-Dorado J, Bravo-Ferrer J for the EURECA project team, et al. EUropean prospective cohort study on Enterobacteriaceae showing REsistance to CArbapenems (EURECA): a protocol of a European multicentre observational study. BMJ Open 2017;7:e015365. doi: 10.1136/bmjopen-2016-015365

The affiliation for author Lul Raka is incorrect. This should read: 'National Institute of Public Health of Kosovo and Faculty of Medicine, University of Prishtina, Prishtina, Kosovo'.

Open Access This is an Open Access article distributed in accordance with the Creative Commons Attribution Non Commercial (CC BY-NC 4.0) license, which permits others to distribute, remix, adapt, build upon this work non-commercially, and license their derivative works on different terms, provided the original work is properly cited and the use is non-commercial. See: http://creativecommons.org/licenses/by-nc/4.0/

(c) Article author(s) (or their employer(s) unless otherwise stated in the text of the article) 2017. All rights reserved. No commercial use is permitted unless otherwise expressly granted.

BMJ Open 2017;7:e015365corr1. doi:10.1136/bmjopen-2016-015365corr1 